\title{
The Global Set-Domination Number in Jump Graphs
}

\author{
N. Pratap Babu Rao \\ VSK University Bellari \\ Dept. of Mathematics \\ SG College Koppal
}

\author{
Swetha N. \\ VSK University Bellari \\ Dept. of Mathsmatics \\ Veersiva College Bellari
}

\begin{abstract}
Let $\mathrm{J}(\mathrm{G})$ be a co-connected jump graph. A set $\mathrm{D} \subset \mathrm{V}(\mathrm{J}(\mathrm{G})-\mathrm{D}$ is a set dominating set (sd-set) if for every $S \subset \mathrm{V}(\mathrm{J}(\mathrm{G})-\mathrm{D}$ there exists a non empty set $\mathrm{T} \subset \mathrm{D}$ such that the sub graph ( $\mathrm{S}$ $U \mathrm{~T})$ is connected. Further $\mathrm{D}$ is a global set dominating set, if $\mathrm{D}$ is an sd-set of both $\mathrm{J}(\mathrm{G})$ and $\mathrm{J}(\bar{G})$. The set domination number $V_{s}$ and the global set domination number $V_{s g}$ of $J(G)$ are defined as expected
\end{abstract}

\section{Keywords}

Set domination, global set domination number

\section{INTRODUCTION}

\section{Theorem 1}

For the tree of order $\mathrm{p}$ with e end vertices $\sqrt{\mathrm{sg}}_{\mathrm{g}}(\mathrm{J}(\mathrm{G}))=\mathrm{p}$-e

\section{Theorem 2}

If diam $\mathrm{J}(\mathrm{G})=3$ then $\sqrt{\mathrm{sg}}_{\mathrm{g}} \mathrm{J}(\mathrm{G}) \leq \sqrt{\mathrm{s}}_{\mathrm{s}}(\mathrm{J}(\mathrm{G}))+2$

If $\operatorname{diam} \mathrm{J}(\mathrm{G}))=4$ then $\sqrt{\mathrm{sg}}_{\mathrm{g}} \mathrm{J}(\mathrm{G}) \leq \sqrt{\mathrm{s}}_{\mathrm{s}}(\mathrm{J}(\mathrm{G}))+1$

If diam $\mathrm{J}(\mathrm{G}) \geq 5$ then $\sqrt{\mathrm{sg}}_{\mathrm{g}} \mathrm{J}(\mathrm{G}) \leq \sqrt{\mathrm{s}}_{\mathrm{s}}(\mathrm{J}(\mathrm{G}))$

Let $\mathrm{J}(\mathrm{G})=(\mathrm{V}, \mathrm{E})$ be a jump graph. A set $\mathrm{D} \subset \mathrm{V}(\mathrm{J}(\mathrm{G})$ is a dominating set of $J(G)$ if every vertex not in $D$ is adjacent to some vertex in D. Further $d$ is a global dominating set of $\mathrm{J}(\mathrm{G})$, if $\mathrm{D}$ is a dominating set of both $\mathrm{J}(\mathrm{G})$ and $\mathrm{J}(\bar{G})$. The domination number $\sqrt{ }(\mathrm{J}(\mathrm{G}))$ of $\mathrm{J}(\mathrm{G})$ is defined similarly the concept of global domination was first introduced by sampathkumar [4] and was also studied by Rall [3] Recently the concept of set domination for a connected graph was introduced by Sampath kumar and L. pushpa latha[ 5]. A set $\mathrm{D} \subset \mathrm{V}(\mathrm{J}(\mathrm{G})$ is an set-dominating set (sd-set)of every set $\mathrm{S} \subset$ $\mathrm{V}(\mathrm{J}(\mathrm{G}))-\mathrm{D}$, there existd a non empty set $\mathrm{T} \subset \mathrm{D}$ such that the sub graph $\langle\mathrm{S} \cup \mathrm{T}\rangle$ induced by $\mathrm{S} \cup$ Tis connected. The setdominating number $\sqrt{s}_{\mathrm{s}} \mathrm{J}(\mathrm{G})=\sqrt{\mathrm{sg}}_{\mathrm{g}}(\mathrm{J}(\mathrm{G})$ ) of jump graph $\mathrm{J}(\mathrm{G})$ is the minimum cardinality of an sd-set. Suppose $J(G)$ is coconnected graph ( i.e, both $\mathrm{J}(\mathrm{G})$ and $\mathrm{J}(\bar{G})$ are connected). The global set domination number $\sqrt{s g}_{\mathrm{sg}}=\sqrt{\mathrm{sg}}_{\mathrm{g}}(\mathrm{J}(\mathrm{G})$ ) of $\mathrm{J}(\mathrm{G})$ is the minimum cardinality of an sd-set of both $\mathrm{J}(\mathrm{G})$ and $\mathrm{J}(\bar{G})$. The purpose of this paper is to initiate a study of $\sqrt{\mathrm{sg}}_{\mathrm{g}}$.

Hence forth we consider only co-connected graph $J(G)$. for a vertex $v \in J(G)$. let $N(v)=\{u: u v \in E\}$ and $N[v]=N(v) U$ $\{\mathrm{v}]$. Also

$$
V_{\mathrm{s}}=V_{\mathrm{sg}}(\mathrm{J}(\mathrm{G})) \text {. }
$$

Since every global sd-set is a global dominating set and $V_{s} \geq 2$ we have $2 \leq \sqrt{ }_{\mathrm{s}} \leq \sqrt{ }_{\mathrm{sg}}$

We observe that for a path $p_{n}$ on $n \geq 4$ vertices $V_{s g}\left(J\left(P_{n}\right)\right)=n-2$ and for a cycle $c_{n}$ on $n \geq 6$ vertices $V_{\mathrm{sg}}\left(J\left(C_{n}\right)\right)=n-3$ when $\sqrt{ }$ ${ }_{\mathrm{sg}}\left(\mathrm{J}\left(\mathrm{C}_{5}\right)\right)=3$.

A $V_{s}$-set is minimum sd-set similarly we define $V_{s g}$-set etc., one can easily determine $\sqrt{\mathrm{sg}}_{\mathrm{g}}$ for a tree.
Theorem 1. In a jump tree $J(T)$ with $p$ vertices and e end vertices that is not a star the set of non-end vertices form a minimum global sd-set and $\sqrt{s g}_{\mathrm{sg}} \mathrm{J}(\mathrm{T})=\mathrm{p}$-e.

Proof: It is known that the set $\mathrm{d}$ of all cut vertices of $\mathrm{T}$ form a $V_{\mathrm{s}}$-set of $\mathrm{T}$ and $V_{\mathrm{s}}=\mathrm{p}-\mathrm{e}$ [5] Clearly the sub graph $\mathrm{V}(\mathrm{J}(\mathrm{T}))$-D in $\mathrm{J}(\bar{T})$ is complete. Since $\mathrm{J}(\mathrm{T}) \neq \mathrm{K}_{1, \mathrm{~m}}$ in $\mathrm{J}(\bar{T})$ each vertex in $\mathrm{V}(\mathrm{J}(\mathrm{T}))-\mathrm{D}$ is adjacent to some vertex in $\mathrm{D}$ this implies that $\mathrm{D}$ is an sd-set of $\mathrm{J}(\mathrm{T})$ also and $\sqrt{s g}_{\mathrm{sg}}=\mathrm{p}-\mathrm{e}$

We now determine some bounds for $\sqrt{ }_{\mathrm{sg}}$.

Theorem 2. Let $\mathrm{J}(\mathrm{G})$ be a co-connected sub graph of order $\mathrm{p} \geq 4$ then

$$
2 \leq \sqrt{\mathrm{sg}}_{\mathrm{g}}(\mathrm{J}(\mathrm{G})) \leq \mathrm{p}-2
$$

Proof: let $u$ and $v$ be adjacent vertices of degree at least two (such vertices clearly exist) Then $\mathrm{V}(\mathrm{J}(\mathrm{G}))-\{\mathrm{u}, \mathrm{v}\}$ is a global sd-set of $\mathrm{J}(\mathrm{G})$ so $\sqrt{\mathrm{sg}}_{\mathrm{g}}(\mathrm{J}(\mathrm{G})) \leq \mathrm{p}-2$.

The bounds in (2) are sharp. The upper bounds attained by paths of length at least 3 and the 5-cycle All jump graphs for which the lower bound is attained can be determined.

Theorem 3: For a jump graph $J(G)$ of order p. $\sqrt{s g}_{\mathrm{sg}}=2$ if and only if

$\operatorname{diam} \mathrm{J}(\mathrm{G})=\operatorname{diam}(\mathrm{J}(\bar{T}))=3$ and either $\mathrm{J}(\mathrm{G})$ or $\mathrm{J}(\bar{T})$ has a bridge which is not an end edge.

Proof; Assume $\sqrt{ }_{\mathrm{sg}}=2$ since $\operatorname{diam} \mathrm{J}(\mathrm{G}) \leq 3$ and $\operatorname{diam}(\mathrm{J}(\bar{G}))$ $\leq 3$ Now, let $\mathrm{D}=\{\mathrm{u}, \mathrm{v}\}$ a $\sqrt{\mathrm{sg}}_{\mathrm{g}}$-set of $\mathrm{J}(\mathrm{G})$ suppose $\mathrm{u}$ and $\mathrm{v}$ are adjacent in $\mathrm{J}(\mathrm{G})$. All vertices in $\mathrm{V}(\mathrm{J}(\mathrm{G}))$-D are adjacent to either $\mathrm{u}$ or $\mathrm{v}$ (but not both). If all such vertices are adjacent to only $\mathrm{u}$ (or v) $\mathrm{G}$ and $\bar{G}$ is disconnected. Hence some vertices of $\mathrm{V}(\mathrm{J}(\mathrm{G})-\mathrm{D}$ are adjacent to $\mathrm{u}$ and some are adjacent to $\mathrm{v}$. If all $\mathrm{x} \in \mathrm{N}(\mathrm{v})-\{\mathrm{u}\}$, hen $\mathrm{x}$ and $\mathrm{y}$ are not adjacent in $\mathrm{J}(\mathrm{G})$,for otherwise

$\{u, v\}$ will not be an sd-set in $J(G)$. Thus uv is a bridge in $\mathrm{J}(\mathrm{G})$ that is not an end edge and $\mathrm{d}(\mathrm{x}, \mathrm{y})=3=\operatorname{diam} \mathrm{J}(\mathrm{G})$ Also in $\mathrm{J}(\bar{G}), \mathrm{d}(\mathrm{u}, \mathrm{v})=3$ and hence $\operatorname{diam} \mathrm{J}(\bar{G})=3$

Conversely, if $\mathrm{J}(\mathrm{G})$ has a bridge uv and is not an end edge and $\operatorname{diam} \mathrm{J}(\mathrm{G})=\operatorname{diam}(\mathrm{J}(\bar{G}))=3$, then every vertex in $\mathrm{J}(\mathrm{G})$ is adjacent to $u$ or to $v$ and hence $\{u, v\}$ is a $\sqrt{s}_{s}$-set in $J(G)$. let $\mathrm{N}_{\mathrm{G}}(\mathrm{u})$ be the set of all neighbors' of $\mathrm{u}$ in $\mathrm{J}(\mathrm{G})$, then $\mathrm{N}_{\mathrm{G}}(\mathrm{u})=$ $N_{G}[v]$ since $u v$ is a bridge in $J(G)$, every vertex of $N_{G}(u)-$ $\{\mathrm{v}\}$ is adjacent to every vertex of $\mathrm{N}_{\mathrm{G}}(\mathrm{u})\{\mathrm{u}\}$ in

$\mathrm{J}(\bar{G})$. Hence $\{\mathrm{u}, \mathrm{v}\}$ is an sd-set of $\mathrm{J}(\bar{G})$ and $\sqrt{\mathrm{sg}}(\mathrm{J}(\mathrm{G}))=2$.

Theorem 4; Let $\mathrm{J}(\mathrm{G})$ be a jump graph with cut vertices. Then

$\left.\sqrt{\mathrm{sg}}_{\mathrm{gg}} \mathrm{J}(\mathrm{G})\right) \leq \bigvee_{\mathrm{s}}(\mathrm{J}(\mathrm{G}))+1=\bigvee_{\mathrm{c}}(\mathrm{J}(\mathrm{G}))+1$ 
Proof: We consider two cases

Case1. There exists a $V_{s}$-set $D$ of $J(G)$ all of whose vertices belong to a single block $B$ of $J(G)$.

Consider a vertex $u \notin D$ such that $u$ is a block $B_{1} \neq$ B let $D^{\prime}=D U\{u\}$ we now show hat $D^{\prime}$ is an sd-set of $J(G)$ let $\mathrm{u}, \mathrm{w} \in \mathrm{V}\left(\mathrm{J}(\mathrm{G})\right.$ ). If $\mathrm{v}, \mathrm{w}$ belongs to a single block $\mathrm{B}_{\mathrm{i}} \neq \mathrm{B}_{1}$ of $\mathrm{J}(\mathrm{G})$ then they are both adjacent to $\mathrm{u}$ in

$\mathrm{J}(\bar{G})$. If $\mathrm{u}$ and $\mathrm{w}$ are in $\mathrm{B}_{1}$ then in $\mathrm{J}(\bar{G})$ both of them are adjacent to a vertex $u_{1} \in D \cap\left(B-B_{1}\right)$ (note that $\sqrt{s}_{s} \geq 2$ ) If $v \in$ $\mathrm{B}_{1}$ and $\mathrm{w} \notin \mathrm{B}_{1}$ then

in $\mathrm{J}(\bar{G}) \mathrm{v}$ is adjacent to $\mathrm{u}_{1}$, and $\mathrm{w}$ is adjacent to $\mathrm{u}$. Further the sub graph $\left\langle\left\{\mathrm{u}, \mathrm{v}, \mathrm{w}, \mathrm{u}_{1}\right\}>\right.$ is connected in $\mathrm{J}(\mathrm{G})$. This proves $D^{\prime}$ is an sd-set of $J(G)$ and

$V_{\mathrm{sg}}(\mathrm{J}(\mathrm{G})) \leq\left|\mathrm{D}^{\prime}\right| \leq \sqrt{\mathrm{s}}_{\mathrm{s}}(\mathrm{J}(\mathrm{G}))+1$.

Case 2. Case 1 is not true.

In this caswe for every $\sqrt{s}_{\mathrm{s}}$-set $\mathrm{D}$ of $\mathrm{J}(\mathrm{G})$ at least two vertices of D belong to different blocks of $J(G)$ one can easily show tha $d$ is also an sd-set of $\mathrm{J} J(\bar{G})$. Hence $\sqrt{s g}_{\mathrm{sg}}(\mathrm{J}(\mathrm{G}))=\sqrt{\mathrm{s}}_{\mathrm{s}} \mathrm{J}(\mathrm{G})(=$ $V_{c}(J(G))$

Theorem 5. Le $\mathrm{J}(\mathrm{G})$ be a jump graph having diameter atleastfive and let $\mathrm{D} \subset \mathrm{V}(\mathrm{J}(\mathrm{G})$. Then $\mathrm{D}$ is a minimal sd-set of $\mathrm{J}(\mathrm{G})$ if and only if $\mathrm{D}$ is a minimal global sd-set of $\mathrm{J}(\mathrm{G})$.

Proof; Suppose D is minimal global sd-set of $J(G)$. let $u$ and $\mathrm{v}$ be such that $\mathrm{d}(\mathrm{u}, \mathrm{v}) \geq 5$. Then $\mathrm{D} \cap \mathrm{N}[\mathrm{u}] \neq \phi$ and $\mathrm{D} \cap \mathrm{N}[\mathrm{v}]$ $\neq \phi$. Let $\mathrm{u}_{1} \in \mathrm{D} \cap \mathrm{N}[\mathrm{u}]$ and

$\mathrm{v}_{1} \in \mathrm{D} \cap \mathrm{N}[\mathrm{v}]$. Since $\mathrm{d}(\mathrm{u}, \mathrm{v}) \geq 5 \mathrm{u}_{1}$ and $\mathrm{v}_{1}$ are non adjacent in $\mathrm{J}(\mathrm{G})$ and hence they are adjacent in $\mathrm{J}(\mathrm{G})$. Also no vertex in $\mathrm{V}(\mathrm{G})-\left\{\mathrm{u}_{1}, \mathrm{v}_{1}\right\}$ is adjacent to both $\mathrm{u}_{1}$ and $\mathrm{v}_{1}$ in $\mathrm{J}(\mathrm{G})$ ssince otherwise $\mathrm{d}(\mathrm{u}, \mathrm{v})^{\lrcorner} \leq 5$
Now in $\mathrm{J}(\bar{G})$, each vertex is adjacent to $\mathrm{u}_{1}$ or $\mathrm{v}_{1}$ (or both) and hence $\left\{\mathrm{u}_{1}, \mathrm{v}_{1}\right\}$ is a connected dominating set of $\mathrm{J}(\bar{G})$, Since every connected dominating set is an sd-set $\left\{\mathrm{u}_{1}, \mathrm{v}_{1}\right\}$ is an sdset of $\mathrm{J}(\bar{G})$,. This proves that $\mathrm{D}$ is an minimal global sd-set of $\mathrm{J}(\mathrm{G})$.

Conversly, If $\mathrm{D}$ is a minimal global sd-set of $\mathrm{J}(\mathrm{G})$ and is noy a minimal sd-set of $J(G)$, then there exists $x \in D$ such that $D$ $\{\mathrm{x}\}$ is also an sd-set of $\mathrm{J}(\mathrm{G})$. As before, if $\mathrm{v}_{1} \in\{\mathrm{D}-\{\mathrm{x}\}\} \cap$ $\mathrm{N}[\mathrm{u}]$ and $\mathrm{v}_{1} \in\{\mathrm{D}-\{\mathrm{x}\}\} \cap \mathrm{N}[\mathrm{v}]$

Then $\left\{\mathrm{u}_{1}, \mathrm{v}_{1}\right\}$ is an sd-set of $\mathrm{J}(\mathrm{G})$ and hence $\mathrm{D}-\{\mathrm{x}]$ is a global sd-set of $J(G)$ a contradiction Hence D is also a minimal sdset of $\mathrm{J}(\mathrm{G})$.

\section{CONCLUSION}

In this paper we studied some characterization of some graphs by global set domination. It can used for further research work on set domination theory.

\section{ACKNOWLEDGEMENT}

Thanks are due to the referee for his comments.

\section{REFERENCES}

[1] Jean Dunbar,R.C Laskar and Ted Monroe,Congr,Numer.85(1991) 67-72

[2] S.T. Hedetniemi and Renu Laskar, in Graph theory and combinatorics,ed B.Bollobas,Academic press London 1984,pp 209-218

[3] D.F. Rall, congr.Numer 80(1991)89-95

[4] E. Sampathkumar, J. Math. Phys.Sci.23(1989)377-385

[5] E. Sampathkumar and L.Pushpa Latha, J. Graph Theory 18(No.1)1994,to appear.

[6] E. Sampathkumar and H.B Walikar J. Math. Phys. Sci 13 (6) (1979), 607-613. 